PROCEEDINGS OF THE

AMERICAN MATHEMATICAL SOCIETY

Volume 133, Number 5, Pages 1417-1422

S 0002-9939(04)07678-6

Article electronically published on November 1, 2004

\title{
LOGARITHMIC COMPARISON THEOREM AND SOME EULER HOMOGENEOUS FREE DIVISORS
}

\author{
F. J. CASTRO-JIMÉNEZ AND J. M. UCHA-ENRÍQUEZ
}

(Communicated by Michael Stillman)

\begin{abstract}
Let $D, x$ be a free divisor germ in a complex manifold $X$ of dimension $n>2$. It is an open problem to find out which are the properties required for $D, x$ to satisfy the so-called Logarithmic Comparison Theorem (LCT), that is, when the complex of logarithmic differential forms computes the cohomology of the complement of $D, x$. We give a family of Euler homogeneous free divisors which, somewhat unexpectedly, does not satisfy the LCT.
\end{abstract}

\section{IntRoduCtion}

It was proved in [2] that if $D$ is a locally quasi-homogeneous free divisor in the complex manifold $X$, then the complex $\Omega^{\bullet}(\log D)$-introduced in 7$]$ - of holomorphic differential forms with logarithmic poles along $D$ calculates the cohomology of the complement of $D$ in $X$. By analogy with Grothendieck's Comparison Theorem ([6]) this fact is summarized by saying that this kind of free divisors satisfies the Logarithmic Comparison Theorem (LCT) or simply that LCT holds for D.

In [1] a partial converse to the result above was proved: if $D$ is a reduced plane curve and LCT holds for $D$, then $D$ is locally quasi-homogeneous 1 and it was shown that, in dimension strictly greater than $2, D$ can verify the LCT without being locally quasi-homogeneous 2 In [1] it has been conjectured that LCT implies Euler homogeneity. The reciprocal is false: consider the divisor

$$
D \equiv f=\left(z\left(x^{4}+y^{5}+x y^{4}\right)=0\right) \subset \mathbf{C}^{3} .
$$

It is Euler homogeneous since $\left(z \partial_{z}\right)(f)=f$ and is easy to prove that it does not satisfy LCT using that the plane curve defined by $x^{4}+y^{5}+x y^{4}=0$ does not either as was proved in [1. It is an open question which Euler homogeneous free divisors do satisfy LCT.

Received by the editors July 21, 2003 and, in revised form, January 8, 2004

2000 Mathematics Subject Classification. Primary 32S20; Secondary 14F10, 32S40.

Key words and phrases. Free divisor, Logarithmic Comparison Theorem, D-modules, Eulerhomogeneous divisor.

This work was partially supported by DGESIC BFM-2001-3164 and FQM-333.

${ }^{1}$ The theorem of K. Saito [7] (for a germ $f$ with isolated singularity, $f$ belongs to its Jacobian ideal is equivalent to the quasi-homogeneity of $f$ ) is strongly necessary in the proof, so the same argument is no longer valid in higher dimensions.

${ }^{2}$ It is the case, for example, for $D \equiv(x y(x+y)(x z+y)=0) \subset \mathbf{C}^{3}$. It is remarkable that this divisor is not analytically a product. 
Let $\mathcal{O}=\mathbf{C}\left\{x_{1}, \ldots, x_{n}\right\}$ be the ring of germs of holomorphic functions around $0 \in \mathbf{C}^{n}$ and $\mathcal{D}$ the ring of differential operators with coefficients in $\mathcal{O}$. Each nonzero element $P$ in $\mathcal{D}$ can be uniquely written in the form $P=\sum_{\alpha} a_{\alpha} \partial^{\alpha}$ where $\alpha=\left(\alpha_{1}, \ldots, \alpha_{n}\right) \in \mathbf{N}^{n}, a_{\alpha} \in \mathcal{O}$ and $\partial^{\alpha}=\partial_{1}^{\alpha_{1}} \cdots \partial_{n}^{\alpha_{n}}$ (here $\partial_{i}$ stands for the partial derivative $\frac{\partial}{\partial x_{i}}$ ). The order of $P$ is by definition the integer number $\max \{|\alpha|=$ $\left.\alpha_{1}+\cdots+\alpha_{n} \mid a_{\alpha} \neq 0\right\}$. A conjecture in this context is the following one:

Conjecture 1.1 (in [9]). LCT holds for $D \Leftrightarrow$ the annihilator $A n_{\mathcal{D}}(1 / h)$ is generated by elements of order 1 , where $h$ is a local equation of $D$.

This conjecture is true for plane curves (see [10, 4] and 8]).

Besides, it has been conjectured that for arrangements of hyperplanes (not necessarily free) LCT holds. The free case is covered in fact in [2] and the case of tame arrangements is covered in [12. In [11] there are some related questions to 1.1] for the case of central arrangements.

So far there are no more general results about the LCT condition. One of the difficulties in the study of this problem is the treatment of the examples. In [3] the authors of this paper proposed computational methods to test LCT, and some partial results - in particular, some experimental backing for 1.1 have been obtained. Nevertheless, the problem for these methods is the limit of the available computer algebra systems for such calculations.

In this work we provide a family of Euler homogeneous free divisors that do not satisfy LCT using an entirely different approach. We take advantage of a necessary condition for the LCT that was introduced in [2] and [1]: if LCT holds, then the morphism

$$
d_{1}: \check{H}^{n-1}(V \backslash 0, \mathcal{O}) \rightarrow \check{H}^{n-1}\left(V \backslash 0, \Omega^{1}(\log D)\right)
$$

is injective, where $V$ is a Stein neighborhood (sufficiently small) of 0 .

\section{The inJeCtivity of $d_{1}$}

Given a germ of a divisor $D \equiv(f=0)$ with $f \in \mathcal{O}$ and following K. Saito [7], the $\mathcal{O}$-module of logarithmic derivations with respect to $D, \operatorname{Der}(-\log D)$, is the set of derivations $\delta$ such that $\delta(f)=m f$ for some $m \in \mathcal{O}$.

We make precise here the last paragraph of the introduction. If $\left\{\omega_{1}, \ldots, \omega_{n}\right\}$ is a free basis of $\Omega^{1}(\log D)$ as $\mathcal{O}_{V}$-module and $\delta_{1}, \ldots, \delta_{n}$ is the dual basis of $\operatorname{Der}(-\log D)$, then

$$
\check{H}^{n-1}\left(V \backslash 0, \mathcal{O}_{\mathbf{C}^{n}}\right) \simeq \check{H}^{n-1}\left(V \backslash 0, \Omega^{0}(\log D)\right)
$$

and

$$
\check{H}^{n-1}\left(V \backslash 0, \mathcal{O}_{\mathbf{C}^{n}}\right)^{n} \simeq \check{H}^{n-1}\left(V \backslash 0, \Omega^{1}(\log D)\right) .
$$

The morphism $d_{1}$ can be read now as

$$
\begin{array}{ccc}
\check{H}^{n-1}\left(V \backslash 0, \mathcal{O}_{\mathbf{C}^{n}}\right) & \stackrel{d_{1}}{\rightarrow} & \check{H}^{n-1}\left(V \backslash 0, \mathcal{O}_{\mathbf{C}^{n}}\right)^{n} \\
{[g]} & \mapsto & \left(\left[\delta_{1} \cdot g\right], \ldots,\left[\delta_{n} \cdot g\right]\right)
\end{array}
$$

since $\check{H}^{n-1}\left(V \backslash 0, \mathcal{O}_{\mathbf{C}^{n}}\right)$ is isomorphic to the space $\mathcal{S}$ of Laurent series, convergent for all $\underline{x}=\left(x_{1}, \ldots, x_{n}\right)$ with $\underline{x} \neq 0$ and whose non-zero coefficients are those with 
strictly negative indices in all variables. For $p \geq n$ we consider the following $\mathbf{C}$ vector spaces of finite dimension:

$$
\begin{aligned}
& G^{p}=\left\{\sum_{\substack{i_{1}, \ldots, i_{n}<0 \\
i_{1}+\cdots+i_{n}=-p}} c_{i_{1} \cdots i_{n}} x_{i}^{i_{1}} \cdots x_{n}^{i_{n}}\right\}, \\
& F^{p}=\left\{\sum_{\substack{i_{1}, \ldots, i_{n}<0 \\
i_{1}+\cdots+i_{n} \geq-p}} c_{i_{1} \cdots i_{n}} x_{i}^{i_{1}} \cdots x_{n}^{i_{n}}\right\} .
\end{aligned}
$$

Clearly $F^{p}=G^{p} \oplus G^{p-1} \oplus \cdots \oplus G^{n}$.

The restriction of $d_{1}$ to each $G^{p}$ is $d_{1 \mid G^{p}}: G^{p} \rightarrow\left(F^{p}\right)^{n}$, and it is defined as

$$
d_{1 \mid G^{p}}([s])=\left(d_{\delta_{1} \mid G^{p}}([s]), \ldots, d_{\delta_{n} \mid G^{p}}([s])\right)=\left(\left[\delta_{1} \cdot s\right], \ldots,\left[\delta_{n} \cdot s\right]\right),
$$

for all $s \in G^{p}$. If $d_{1}$ is injective, so is its restriction to each $G^{p}$, and this is the core of our method: take successively for each $p \geq n$ the corresponding matrix of $d_{1 \mid G^{p}}$ and check if it is injective.

It is important to point out that if in a basis of $\operatorname{Der}(-\log D)$ there exists a derivation of the form $\sum w_{i} x_{i} \partial_{i}$ with $w_{i} \geq 0$ and at least one of the $w_{i}$ is nonzero, then the morphism $d_{1 \mid G^{p}}$ is injective. Although, for plane curves, $d_{1}$ injective and LCT are equivalent conditions (see [1]), this fact is false in higher dimensions: consider again the divisor $D \equiv\left(z\left(x^{4}+y^{5}+x y^{4}\right)=0\right)$ that has the derivation $z \partial_{z}$ in a suitable basis of $\operatorname{Der}(-\log D))$.

\section{A NON-Euler homogeneous EXAMPle}

In this section we illustrate the use of the injectivity of $d_{1}$ via an example. We study a non-Euler homogeneous divisor $(f=0)$ in $\mathbf{C}^{3}$, that is, for which there doesn't exist a logarithmic derivation such that $\delta(f)=f$. It does not satisfy the LCT and could be considered as a consequence of the behaviour of the Reiffen plane curves (see 4]). Its interest lies in the difficulty of treating this example with the available systems with the methods of [3], due to the size of the Gröbner bases computations required.

The divisor is $D \equiv\left(f=(x z+y)\left(x^{4}+y^{5}+x y^{4}\right)=0\right) \subset \mathbf{C}^{3}$. It is free as a consequence of Saito's criterion (see [7]) applied to the following basis of $\operatorname{Der}(-\log D)$ :

$$
\begin{aligned}
\delta_{1}= & (x z+y) \partial_{z}, \\
\delta_{2}= & \left(8 x^{2}+10 x y\right) \partial_{x}+\left(6 x y+8 y^{2}\right) \partial_{y}+(-2 y z+2 y) \partial_{z}, \\
\delta_{3}= & \left(x y^{2}+\frac{1}{4} y^{3}+\frac{5}{4} x^{2}-\frac{25}{4} x y\right) \partial_{x} \\
& +\left(\frac{3}{4} y^{3}-\frac{1}{4} x^{2}+\frac{5}{4} x y-5 y^{2}\right) \partial_{y} \\
& +\left(\frac{1}{4} y^{2} z^{2}-\frac{1}{4} y^{2} z+\frac{5}{4} y z+\frac{1}{4} x\right) \partial_{z},
\end{aligned}
$$

whose determinant (of coefficients with respect to $\partial_{1}, \partial_{2}, \partial_{3}$ ) is $-2 \cdot f$. It is not Euler homogeneous since the ideal generated by the $a_{i}$ where $\delta_{i}(f)=a_{i} f$ (where $\left.a_{1}=x, a_{2}=40 x+48 y, a_{3}=\frac{1}{4} y^{2} z+\frac{19}{4} y^{2}+\frac{25}{4} x-30 y\right)$ is contained in the ideal generated by $x, y, z$. 
We show that $d_{1}$ is not injective, because it is not injective on $G^{3}$. The only element we have to check is $\frac{1}{x y z}$. We have

$$
\begin{aligned}
\delta_{1}\left(\frac{1}{x y z}\right)= & (x z+y)\left(\frac{-1}{x y z^{2}}\right)=0 \\
\delta_{2}\left(\frac{1}{x y z}\right)= & \left(8 x^{2}+10 x y\right)\left(\frac{-1}{x^{2} y z}\right)+\left(6 x y+8 y^{2}\right)\left(\frac{-1}{x y^{2} z}\right) \\
& +(-2 y z+2 y)\left(\frac{-1}{x y z^{2}}\right)=0, \\
\delta_{3}\left(\frac{1}{x y z}\right)= & \left(x y^{2}+\frac{1}{4} y^{3}+\frac{5}{4} x^{2}-\frac{25}{4} x y\right)\left(\frac{-1}{x^{2} y z}\right) \\
& +\left(\frac{3}{4} y^{3}-\frac{1}{4} x^{2}+\frac{5}{4} x y-5 y^{2}\right)\left(\frac{-1}{x y^{2} z}\right) \\
& +\left(\frac{1}{4} y^{2} z^{2}-\frac{1}{4} y^{2} z+\frac{5}{4} y z+\frac{1}{4} x\right)\left(\frac{-1}{x y z^{2}}\right)=0 .
\end{aligned}
$$

This study can be extended (although it is a little tedious!) to the family of divisors $D_{p, q} \equiv\left((x z+y)\left(x^{p}+y^{q}+x y^{q-1}\right)=0\right) \subset \mathbf{C}^{3}$ for $4 \leq p<q$ or even in any dimension considering $D_{p, q} \equiv\left(\left(x_{1}^{p}+x_{2}^{q}+x_{1} x_{2}^{q-1}\right)\left(\prod_{i=3}^{n}\left(x_{1} x_{i}+x_{2}\right)\right)=0\right), 4 \leq p<q$, as we will do in the next section for a more interesting family.

\section{A family With "Euler vector fields"}

Here we present a family of Euler homogeneous free divisors that do not satisfy the LCT. In our opinion it is an interesting family because:

- They have not been constructed starting with non-quasi-homogeneous plane curves but from quasi-homogeneous cuspids.

- They have in their bases of logarithmic derivations an Euler vector field in a broad sense of the word: there exists a vector field $E=\sum_{i=1}^{n} w_{i} x_{i} \partial_{i}, w_{i} \in$ Q, with $E(f)=f$, where $f=0$ is a local equation of the divisor.

So let us consider the family $\mathcal{F}$ of divisors

$$
D_{p, q} \equiv\left(f_{p, q}=\left(x_{1}^{p}-x_{2}^{q}\right) \prod_{i=3}^{n}\left(x_{1} x_{i}+x_{2}\right)=0\right) \subset \mathbf{C}^{n}
$$

with $p<q$. We will prove that

- Every element of $\mathcal{F}$ is an Euler homogeneous free divisor.

- For a (infinite) subfamily $\mathcal{E}$ of $\mathcal{F}, d_{1}$ is not injective, so LCT does not hold.

Proposition 4.1. The elements of $\mathcal{F}$ are (Euler homogeneous) free divisors.

Proof. By Saito's criterion, [7], it is enough to show a collection of $n$ elements in $\operatorname{Der}\left(-\log D_{p, q}\right)$ whose determinant (of the coefficients of the derivations $\partial_{1}, \ldots, \partial_{n}$ ) is equal to $u \cdot f_{p, q}$ where $u$ is a polynomial such that $u(0) \neq 0$. We take

$$
\begin{aligned}
\delta_{1}= & q x_{1} \partial_{1}+p x_{2} \partial_{2}+\sum_{i=3}^{n}(p-q) x_{i} \partial_{i}, \\
\delta_{2}= & \left(\frac{q}{p(q-p)} x_{1} x_{2}^{q-2} x_{n}+\frac{1}{p} x_{2}^{q-1}\right) \partial_{1} \\
& +\left(\frac{1}{q-p} x_{2}^{q-1} x_{n}+\frac{1}{q} x_{1}^{p-1}\right) \partial_{2} \\
& +\sum_{i=3}^{n-1}\left(\frac{1}{p} x_{2}^{q-2} x_{i}^{2}-\frac{1}{p} x_{2}^{q-2} x_{i} x_{n}-\frac{1}{q} x_{1}^{p-2}\right) \partial_{i}-\frac{1}{q} x_{1}^{p-2} \partial_{n}, \\
\delta_{i}= & \left(x_{1} x_{i}+x_{2}\right) \partial_{i} \text { for } i=3, \ldots, n .
\end{aligned}
$$

The corresponding determinant is equal to $f_{p, q}$. 
Note that $\delta_{1}$ is an Euler vector field with negative weights for $x_{3}, \ldots, x_{n}$ and positive for $x_{1}, x_{2}$.

Theorem 4.2. If $q+p+\sum_{i=3}^{n}(p-q) \alpha_{i}=0, \alpha_{i}>0$ and $3 \leq p<q$, then

$$
d_{1}\left(\frac{1}{x_{1} x_{2} x_{3}^{\alpha_{3}} \cdots x_{n}^{\alpha_{n}}}\right)=0 \text { in } \check{H}^{n-1}\left(V \backslash 0, \mathcal{O}_{\mathbf{C}^{n}}\right)^{n} .
$$

So $d_{1}$ is not injective and LCT does not hold.

Proof. First, it is clear that

$$
\delta_{1}\left(\frac{1}{x_{1} x_{2} x_{3}^{\alpha_{3}} \cdots x_{n}^{\alpha_{n}}}\right)=\left(q+p+\sum_{i=3}^{n}(p-q) \alpha_{i}\right) \frac{1}{x_{1} x_{2} x_{3}^{\alpha_{3}} \cdots x_{n}^{\alpha_{n}}}=0
$$

by the diophantine hypothesis on $p, q$ and the $\alpha_{i}$.

Second we have

$$
\begin{gathered}
\delta_{i}\left(\frac{1}{x_{1} x_{2} x_{3}^{\alpha_{3}} \cdots x_{i}^{\alpha_{i}} \cdots x_{n}^{\alpha_{n}}}\right) \\
=-\alpha_{i}\left(\frac{x_{1} x_{i}}{x_{1} x_{2} x_{3}^{\alpha_{3}} \cdots x_{i}^{\alpha_{i}+1} \cdots x_{n}^{\alpha_{n}}}+\frac{x_{2}}{x_{1} x_{2} x_{3}^{\alpha_{3}+1} \cdots x_{n}^{\alpha_{n}}}\right)=0,
\end{gathered}
$$

for $i=3, \ldots, n$.

Finally,

$$
\begin{aligned}
& \delta_{2}\left(\frac{1}{x_{1} x_{2} x_{3}^{\alpha_{3}} \cdots x_{n}^{\alpha_{n}}}\right) \\
& =\left(\frac{q}{p(q-p)} x_{1} x_{2}^{q-2} x_{n}+\frac{1}{p} x_{2}^{q-1}\right) \frac{-1}{x_{1}^{2} x_{2} x_{3}^{\alpha_{3} \cdots x_{n}^{\alpha}}} \\
& +\left(\frac{1}{q-p} x_{2}^{q-1} x_{n}+\frac{1}{q} x_{1}^{p-1}\right) \frac{-1}{x_{1} x_{2}^{2} x_{3}^{\alpha_{3} \cdots x_{n}^{\alpha}}} \\
& +\left(\sum_{i=3}^{n-1} \frac{1}{p} x_{2}^{q-2} x_{i}^{2}-\frac{1}{p} x_{2}^{q-2} x_{i} x_{n}-\frac{1}{q} x_{1}^{p-2}\right) \frac{-1}{x_{1} x_{2} x_{3}^{\alpha_{3}} \cdots x_{i}^{\alpha_{i}+1} \cdots x_{n}^{\alpha_{n}}}
\end{aligned}
$$

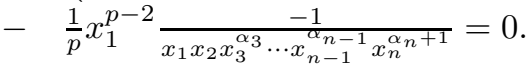

The key is again that we have eliminated $x_{1}$ or $x_{2}$ of every Laurent monomial so that it becomes 0 in $\check{H}^{n-1}\left(V-0, \mathcal{O}_{\mathbf{C}^{n}}\right)$.

Remark 4.3. We have also studied the case $p=2<q$, that is, the family of divisors $D_{2, q} \equiv\left(f_{2, q}=\left(x_{1}^{2}-x_{2}^{q}\right) \prod_{i=3}^{n}\left(x_{1} x_{i}+x_{2}\right)=0\right) \subset \mathbf{C}^{n}$ for many values of $q$, and they satisfy LCT: it is apparent that this fact is true for any $q>2$, but we do not have any proof of this result. In all the examples, we have obtained that the annihilator ideal $A n n_{\mathcal{D}}\left(1 / f_{2, q}\right)$ is generated by elements of order 1 , and then we use the Duality Theorem of $[5]$.

We would like to point out that for $p=2<q$ the corresponding divisor is a product (analytically): it is a consequence of the presence of the derivation $\delta_{2}=$ $\cdots-\frac{1}{2} \partial_{n}$. A different approach to prove LCT for $D_{2, q}$, for $n=3$, could be to find an analytical change of variables producing a weighted homogeneous equation in 2 variables.

\section{Conclusions}

We have provided a family of Euler homogeneous free divisors (in any dimension greater than 2) - defined by polynomials that are "weakly weighted homogeneous" with some negative weights - that do not satisfy LCT. It is an interesting question whether this set of weights is valid locally around 0 for this family, that is, if for 
any point $x$ in a small enough neighborhood of 0 the divisor $(D, x)$ admits a local weighted homogeneous equation $h$ with eventually some negative weights.

\section{ACKNOWLEDGEMENTS}

We deeply thank Prof. David Mond for all his suggestions and patience during the elaboration of this work. We are also grateful to the referee for his comments and advice.

\section{REFERENCES}

[1] Calderón-Moreno F.J., D. Mond, L. Narváez-Macarro and F.J. Castro-Jiménez. Logarithmic Cohomology of the Complement of a Plane Curve. Comment. Math. Helv. 77 (2002), no. 1, 24-38. MF,1898392 (2003e:32047)

[2] Castro-Jiménez, F.J., Mond, D. and Narváez-Macarro, L. Cohomology of the complement of a free divisor. Trans. Amer. Math. Soc. 348 (1996), no. 8, 3037-3049. MR.1363009 (96k:32072)

[3] Castro-Jiménez, F.J., Ucha-Enríquez, J.M. Testing the Logarithmic Comparison Theorem In preparation.

[4] Castro-Jiménez, F.J., Ucha-Enríquez, J.M. Explicit comparison theorems for D-modules. J. Symbolic Comput., Special Issue on Effective Methods in Rings of Differential Operators, 32 (2001) no. 6, 677-685. MR.1866710 (2003m:16034)

[5] Castro-Jiménez, F. J. and J. M. Ucha-Enríquez. Free Divisors and Duality for D-Modules Proc. Steklov Inst. of Math., volume 238, pages 88-96. 2002. MR1969307 (2004a:32017)

[6] Grothendieck, A. On the de Rham cohomology of algebraic varieties. Publ. Math. de l'I.H.E.S. 29 (1966), 95-103. MR 0199194 (33:7343)

[7] Saito, K. Theory of logarithmic differential forms and logarithmic vector fields. J. Fac. Sci. Univ. Tokyo 27:256-291, 1980. MR0586450 (83h:32023)

[8] Torrelli, T. Equations fonctionnelles pour une fonction sur un espace singulier. $\mathrm{Ph}$. D. Thesis, 1998.

[9] Torrelli, T. Sur les germes de fonctions méromorphes définis par un système differentiel d'ordre 1. Preprint, 2002

[10] Ucha-Enríquez, J.M. Métodos constructivos en álgebras de operadores diferenciales. Ph. D. Thesis, Universidad de Sevilla, 1999.

[11] Walther, U. Bernstein-Sato polynomial versus cohomology of the Milnor fiber for generic arrangements of hyperplanes Preprint math.AG/0204080.

[12] Wiens, J. and Yuzvinsky, S. De Rham Cohomology of logarithmic forms on arrangements of hyperplanes. Trans. Amer. Math. Soc. 349 (1997) no. 4, 1653-1662. MR1407505 (97h:52013)

Departamento de Álgebra, Facultad de Matemáticas, Universidad de Sevilla, Apdo 1160, E-41080 Sevilla, Spain

E-mail address: castro@us.es

Departamento de Álgebra, Facultad de Matemáticas, Universidad de Sevilla, Apdo 1160, E-41080 Sevilla, Spain

E-mail address: ucha@us.es 